\title{
QUANTUM TUNNELLING OF MAGNETIZATION IN UNIAXIAL MAGNETIC CLUSTERS
}

\author{
V.V. Dobrovitski, E.R. Rakimetov \\ Moscow State University, Physics Dept. \\ Vorobievy gory, Moscow 119899, Russia \\ B. Barbara \\ CNRS - Laboratoire Louis Néel \\ 25, Av. des Martyrs, 38042, BP166, Grenoble, Cedex 9, France \\ AND A.K. ZvEZDIN \\ Institute of General Physics of Russian Academy of Sciences \\ Vavilova st., 38, Moscow 117942, Russia
}

In the present paper the uniaxial cluster, such as $\mathrm{Mn}_{12}$, is considered. For explanation of magnetization tunnelling in such a system, we propose to account for vibron excitations of $\mathrm{Mn}_{12}$ cluster; the presence of vibron can reduce the symmetry of the system and create the second-order anisotropy in the basal plane, necessary for tunnelling. Using the fact that the vibron frequency is low in comp arison with magnetic moment precession rate, we calculated the rate of magnetization tunnelling within WKB-approximation using the instanton techniques. The exact analytic instanton solution was obtained for arbitrary values of the external field, uniaxial and transversal anisotropy. We discuss also the mechanisms of quantum oscillations of the parameters of tunnelling.

PACS numbers: $75.45 .+\mathrm{j}, 75.50 . \mathrm{Tt}$

The problem of macroscopic quantum tunnelling (MQT) of magnetization attracts a great interest now. Numerous attempts of experimental investigation of this phenomenon were undertaken in recent years [1-3]. But the serious problem of unambiguous interpretation exists: it is known [4] that under certain conditions the variation of tunnelling parameters (e.g., particles' sizes) makes the thermoactivated regime very similar to the $\mathrm{MQT}$ regime.

From this point of view the cluster systems, such as $\mathrm{Mn}_{12} \mathrm{O}_{12}\left(\mathrm{O}_{2} \mathrm{CR}\right)_{16}\left(\mathrm{H}_{2} \mathrm{O}\right)_{4}$ (usually denoted as $\mathrm{Mn}_{12} \mathrm{Ac}$ ) could be useful. Crystals of such compounds have tetragonal symmetry and consist of organic molecules linked by relatively weak Van der Waals forces; each molecule contains a cluster of 12 magnetic ions of Mn which are coupled by strong exchange interaction [5]. The cluster possesses 
the strong magnetic anisotropy of the "easy axis" type, so that the magnetic relaxation in such crystals, to a high degree of accuracy, can be considered as a relaxation in the system of independent superparamagnetic particles of the same size.

But in such systems the problem of low-temperature magnetic relaxation appears; an interpretation of relaxation in terms of MQT is problematic: according to the general theory [6], the tunnelling probability is zero for a uniaxial quantum system since the projection of a mechanic moment onto a symmetry axis $J_{z}$ is conserved. The possible fourth-order anisotropy in the basal plane is extremely weak [7] and cannot solve the problem.

Nevertheless, there is a way for appearing of the second-order anisotropy in the basal plane: if the ground state of the electron subsystem is degenerated, the Jahn-Teller instability can develop [8], mixing the motion of nuclear subsystem with the motion of electron subsystem and producing the so-called vibron states. Therefore, on the average the system is tetragonal, though the instant configuration has lower symmetry; the presence of a rhombic anisotropy in the basal plane breaks down the conservation of $J_{z}$ and permits the tunnelling.

The aim of this paper is to study the effects of spin-vibron interaction in the MQT and main features in such system basing on the simple enough but quite realistic model.

For definiteness, we consider normal oscillations of tetragonal system which deform the square, lying in the basal plane into the rectangle or rhomb $\left(B_{1}\right.$ or $B_{2}$, according to [8]). The corresponding normal coordinate will be denoted as $\Delta$. We will describe the magnetization vector by spherical angles $\theta$ and $\phi$. The former is measured from tetragonal $c$-axis (easy axis), and the latter - from the axis of oscillations lying in the basal plane. Let us assume $\theta=0$ at initial time; the external applied field is directed along $\theta=\pi$. We will calculate the probability of tunnelling from the metastable minimum $\theta=0$ into the stable one $\theta=\pi$. The tunnelling probability $\Gamma$ can be calculated within the WKB-approximation using the instanton approach based on the formalism of path integrals in imaginary time $\tau[6]$, in this case

$$
\Gamma=A \exp \left(-S_{\mathrm{cl}} / \hbar\right),
$$

where $S_{\mathrm{cl}}$ is the action along the instanton trajectory, which starts at $\tau \rightarrow-\infty$ in the point $\theta=0$, at $\tau=0$ passes through the turning point and at $\tau \rightarrow+\infty$ goes back to the point $\theta=0$. The Lagrangian of the magnetic moment interacting with the vibron oscillation will have the following form (in imaginary time):

$$
\begin{aligned}
L= & \mathrm{i} \frac{M}{\gamma} \dot{\phi}(1-\cos \theta)+K \sin ^{2} \theta+M H(\cos \theta-1)+l \Delta \sin ^{2} \theta \sin ^{2} \phi \\
& +\frac{m \dot{\Delta}^{2}}{2}-\beta|\Delta|+\frac{\kappa \Delta^{2}}{2}+\frac{\beta^{2}}{2 \kappa},
\end{aligned}
$$

where $M$ is the magnetic moment of the cluster, $K$ is the easy-axis anisotropy constant, $H$ is the external field, $l$ is the parameter describing the coupling between the molecule deformation and transversal anisotropy induced by this deformation, $\kappa$ is the stiffness constant corresponding to the given mode of oscillations, $m$ is the effective mass of this mode, and $\beta$ is the parameter of linear vibronic interaction, 
characterizing the variation of potential energy of deformed system $(\Delta \neq 0)$ from the energy value corresponding to tetragonally-symmetric nuclear configuration. $(\Delta=0)$.

The vibron oscillation, due to its large effective mass, is much slower than the motion of the magnetic moment, and we have a natural small parameter: the ratio of the moment precession period and vibron oscillation period.

Zeroth-order equations for the magnetic moment motion, when $\Delta$ can be taken as constant, have an exact analytic instanton solution

$$
\begin{aligned}
& \tan \theta^{0} / 2=\left(\frac{\omega_{K}-\omega_{H}}{\omega_{H}} \frac{\omega_{1}}{\omega_{1}+\omega_{\Delta} \operatorname{sh}^{2} x}\right)^{1 / 2}, \\
& \sin \phi^{0}=\mathrm{i}\left[\frac{\left(\omega_{K}-\omega_{H}\right) \operatorname{sh}^{2} x}{\omega_{1}+\omega_{\Delta} \operatorname{sh}^{2} x}\right]^{1 / 2},
\end{aligned}
$$

where $\omega_{K}=2 \gamma K / M, \omega_{H}=\gamma H, \omega_{\Delta}=2 \gamma \Delta l / M, \omega_{1}=\omega_{K}-\omega_{H}+\omega_{\Delta}$, and $x=\tau \sqrt{\left(\omega_{K}-\omega_{H}\right) \omega_{1}}$ is the dimensionless variable which is proportional to the imaginary time $\tau$.

The vibron energy minima are located at $\Delta_{0}= \pm \beta / \kappa$; the choice of the minimum defines the choice of boundary conditions. Without the loss of generality we choose $\Delta_{0}=\beta / \kappa$, and $\Delta(\tau \rightarrow \pm \infty)=\Delta_{0}$. Then the vibron motion can be determined

$$
\begin{aligned}
\Delta= & \Delta_{0}-\frac{l P}{2 m \omega_{0}} \exp \left(-\omega_{0}|\tau|\right), \\
P= & -\frac{1-\epsilon}{\omega_{K}} \frac{4}{(1+\delta)^{3 / 2}}\left[\frac{\sqrt{\epsilon(\epsilon+\delta)(1+\delta)}}{\delta(1-\epsilon)}\right. \\
& \left.-\frac{1}{2} \ln \frac{\epsilon+\delta+\epsilon(1+\delta)+\sqrt{\epsilon(\epsilon+\delta)(1+\delta)}}{\epsilon+\delta+\epsilon(1+\delta)-\sqrt{\epsilon(\epsilon+\delta)(1+\delta)}}\right],
\end{aligned}
$$

where $\omega_{0}^{2}=\kappa / m, \epsilon=\left(\omega_{K}-\omega_{H}\right) / \omega_{K}$, and $\delta=\omega_{\Delta} / \omega_{K}$. It is worth noting that the value of $\Delta$ can be significant enough at relatively small $\omega_{0}$.

Using (3),(4) and (5), we obtain the total instanton action in zeroth-order approximation (in (3) and (4) the value $\Delta=\Delta(\tau=0)$ should be used):

$$
\begin{aligned}
S_{\mathrm{cl}}= & S_{\mathrm{m}}^{0}+\frac{l^{2} P^{2}}{4 m \omega_{0}}, \\
S_{\mathrm{m}}^{0}= & \frac{M}{\gamma}\left[\ln \frac{2 \epsilon+\delta+2 \sqrt{\epsilon(\epsilon+\delta)}}{2 \epsilon+\delta-2 \sqrt{\epsilon(\epsilon+\delta)}}\right. \\
& \left.-\frac{1-\epsilon}{\sqrt{1+\delta}} \ln \frac{2 \epsilon+(1+\epsilon) \delta+2 \sqrt{\epsilon(\epsilon+\delta)(1+\delta)}}{2 \epsilon+(1+\epsilon) \delta-2 \sqrt{\epsilon(\epsilon+\delta)(1+\delta)}}\right] .
\end{aligned}
$$

Substituting this result into (1), we obtain the tunnelling rate. 
Thus, we explicitly showed that the interaction of the magnetic moment with the oscillations of the nuclear subsystem (or with vibron oscillations) in axially-symmetric system such as the cluster $\mathrm{Mn}_{12}$ can open the way for the tunneling of the magnetic moment.

\section{References}

[1] J. Tejada, X.X. Zhang, L. Balcells, J. Appl. Phys. 73, 6709 (1993).

[2] B. Barbara, L.C. Sampaio, J.E. Wegrowe, B.A. Ratnam, A. Marchand, C. Paulsen, M.A. Novak, J.L. Tholence, M. Uehara, D. Fruchart, J. Appl. Phys. 73, 6703 (1993).

[3] D.D. Awschalom, J.F. Smyth, G. Grinstein, D.P. DiVincenzo, D. Loos, Phys. Rev. Lett. 68, 3092 (1992) and 71, 4279(E) (1993).

[4] B. Barbara, L. Gunther, J. Magn. Magn. Mater. 128, 35 (1993).

[5] R. Sessoli, II.L. Tsai, A.R. Schake, S. Wang, J.B. Vincent, K. Folting, D. Gatteschi, G. Christou, D.N. Hendrickson, J. Am. Chem. Soc. 115, 1804 (1993).

[6] P.C.E. Stamp, E.M. Chudnovski, B. Barbara, Int. J. Mod. Phys. B 6, 1355 (1992).

[7] J. Villain, F. Iartman-Boutron, R. Sessoli, A. Rettori, Europhys. Lelt. 27, 159 (1994).

[8] I.B. Bersuker, V.Z. Polinger, Vibron Inleractions in Molecules and Crystals, Nauka, Moskva 1983 (in Russian). 